\title{
Three-dimensional polycaprolactone scaffold-conjugated bone morphogenetic protein-2 promotes cartilage regeneration from primary chondrocytes in vitro and in vivo without accelerated endochondral ossification
}

\author{
Claire G. Jeong, ${ }^{1}$ Huina Zhang, ${ }^{1}$ Scott J. Hollister ${ }^{1,2,3}$ \\ ${ }^{1}$ Department of Biomedical Engineering, University of Michigan, Ann Arbor, Michigan 48109-2125 \\ ${ }^{2}$ Department of Mechanical Engineering, University of Michigan, Ann Arbor, Michigan 48109-2125 \\ ${ }^{3}$ Department of Surgery, The University of Michigan, Ann Arbor, Michigan 48109-0329 \\ Received 16 August 2011; accepted 30 August 2011 \\ Published online 21 May 2012 in Wiley Online Library (wileyonlinelibrary.com). DOI: 10.1002/jbm.a.33249
}

\begin{abstract}
As articular cartilage is avascular, and mature chondrocytes do not proliferate, cartilage lesions have a limited capacity for regeneration after severe damage. The treatment of such damage has been challenging due to the limited availability of autologous healthy cartilage and lengthy and expensive cell isolation and expansion procedures. Hence, the use of bone morphogenetic protein-2 (BMP-2), a potent regulator of chondrogenic expression, has received considerable attention in cartilage and osteochondral tissue engineering. However, the exact role of BMP-2 in cartilage repair has been postulated to promote both cartilage formation and subsequent cartilage degradation through hypertrophy and endochondral ossification. Furthermore, it is likely that the manner in which BMP-2 is presented to chondrocytes will influence the physiologic pathway (repair vs. degeneration). This study investigates the relative influence of BMP-2 on cartilage matrix and potential subsequent bone matrix production using primary chondrocytes seeded on designed 3D polycap-
\end{abstract}

rolactone (PCL) scaffolds with chemically conjugated BMP-2. The results show that chemically conjugated BMP-2 PCL scaffolds can promote significantly greater cartilage regeneration from seeded chondrocytes both in vitro and in vivo compared with untreated scaffolds. Furthermore, our results demonstrate that the conjugated BMP-2 does not particularly accelerate endochondral ossification even in a readily permissible and highly vascular in vivo environment compared with untreated PCL scaffolds. This study not only reveals the potential use of the BMP-2 conjugation delivery method for enhanced cartilage tissue formation but also gives new insights for the effects of conjugated BMP-2 on cartilage regeneration and osteochondral ossification. (C) 2012 Wiley Periodicals, Inc. J Biomed Mater Res Part A: 100A: 2088-2096, 2012.

Key Words: BMP-2 conjugation, cartilage regeneration, endochondral ossification, primary chondrocytes, 3D polycaprolactone scaffold

How to cite this article: Jeong CG, Zhang H, Hollister SJ. 2012. Three-dimensional polycaprolactone scaffold-conjugated bone morphogenetic protein-2 promotes cartilage regeneration from primary chondrocytes in vitro and in vivo without accelerated endochondral ossification. J Biomed Mater Res Part A 2012:100A:2088-2096.

\section{INTRODUCTION}

Degenerative cartilage disease remains a major cause of disability with over 1 million individuals in the US alone annually treated for articular cartilage lesions. ${ }^{1}$ These lesions can affect the cartilage alone (chondral lesions) or the bone and cartilage together (osteochondral lesions). Although microfracture, autologous cell implantation (ACI), and osteoarticular transfer system are widely used therapies to treat these lesions, they all have significant limitations, and thus the search continues for therapies that can robustly address chondral and osteochondral lesions.

One widely studied potential therapy is the use of bone morphogenetic protein-2 (BMP-2). BMP-2 is known to regulate and promote proliferation, differentiation, and apoptosis of various cell lineages in both bone and cartilage development and homeostasis. ${ }^{2-6}$ Recently, van der Kraan et al. ${ }^{7}$ postulated that bone morphogenetic proteins (BMPs) may play two contradictory roles (both protective and harmful) in cartilage development and osteoarthritis development and progression. For bone and cartilage reconstruction, BMP-2 is often injected locally and directly ${ }^{8}$ or delivered after adsorption on various biomaterials such as collagen sponges, ${ }^{9,10}$ hydrogels, ${ }^{10-13}$ hydroxyapatite, ${ }^{14}$ and biomaterial based microspheres. ${ }^{15,16}$

Physical adsorption provides limited binding, leading to bolus delivery that requires high doses to be biologically effective. The initial burst release characteristic of many physical adsorption techniques uncontrolled BMP-2 

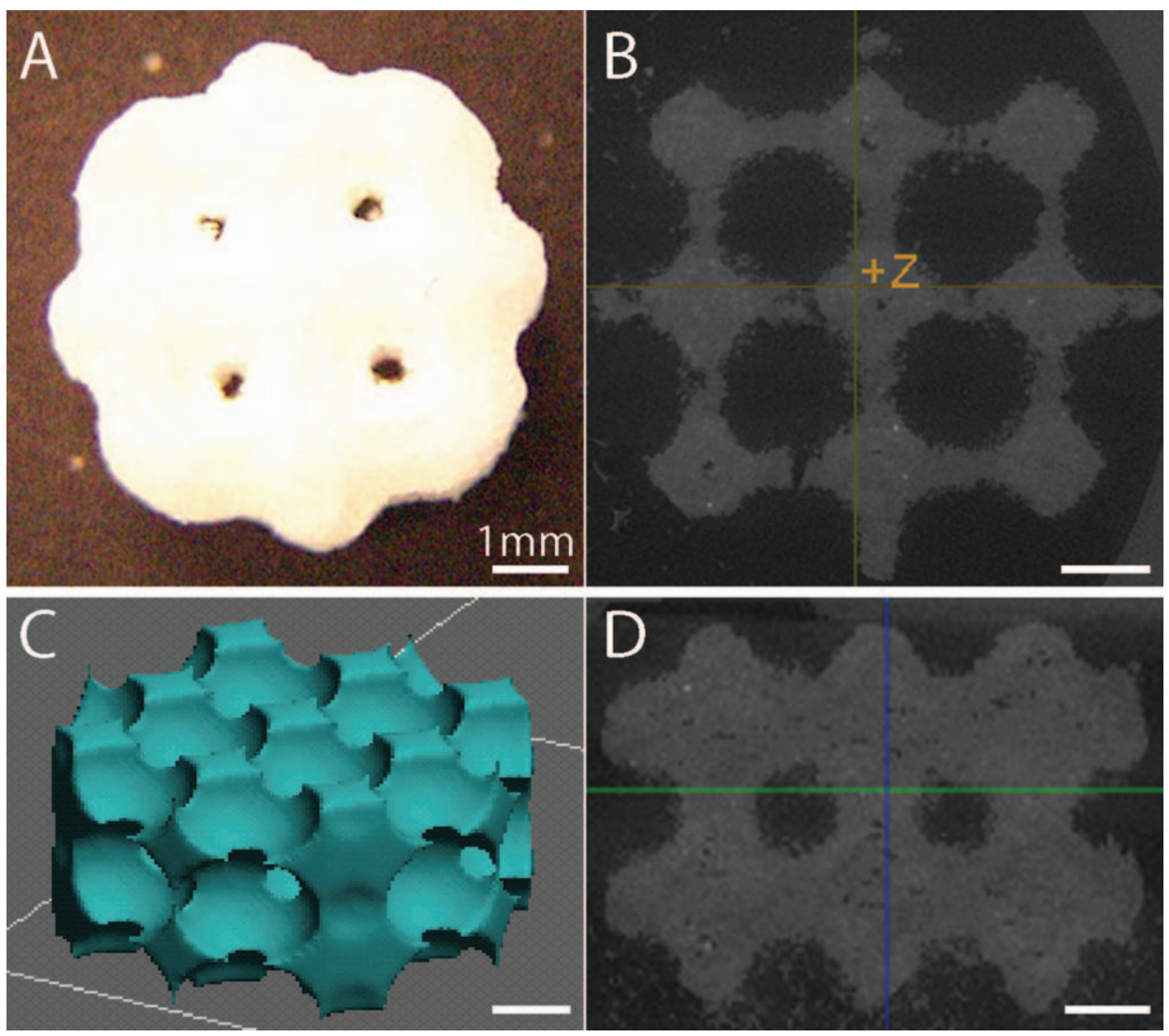

FIGURE 1. Scaffold design: (A) a digital picture, (B) micro-CT image of a 3D PCL scaffold from the top view (the center slice), (C) 3D isometric view of scaffold design (.STL file image), and (D) micro-CT image of a 3D PCL scaffold from the side view. [Color figure can be viewed in the online issue, which is available at wileyonlinelibrary.com.]

diffusion into surrounding tissue space and undesired tissue formation. Previously, our group has successfully introduced a method of directly attaching BMP-2 to designed, threedimensional (3D) polycaprolactone (PCL) scaffolds by a crosslinking conjugation. Conjugation not only retained BMP-2 locally in 3D space but also maintained a higher rate of bioactivity as measured by phosphorylated smad pathway activation expression compared with physical adsorption. ${ }^{17}$ Even though BMP-2 has been used extensively to enhance new bone formation, it is also known to stimulate prechondrocyte condensation, and proliferation, matrix production, and aggrecan synthesis by mature chondrocytes. ${ }^{7,18}$ The aim of this study was therefore to evaluate the effect of chemically conjugated BMP-2 PCL scaffolds on chondrogenesis by primary chondrocytes in vitro and in a subcutaneous in vivo model to determine if controlled BMP-2 delivery is effective for cartilage regeneration. Furthermore, we wanted to examine whether BMP-2 could protect formed cartilage or whether it would accelerate endochondral ossification, the question raised by van de Kraan et al. $^{7}$ To provide the most challenging environment to test the chondroprotective properties of BMP-2, we implanted scaffold/chondrocytes constructs with or without conjugated BMP-2 into a vascularized subcutaneous environment after cartilage was initially formed in vitro (Fig. 1).

\section{RESULTS}

Chemically conjugated BMP-2 PCL scaffolds produced and maintained significantly more cartilage matrix quantified by the amount of sulfated glycosaminoglycans (sGAG) formed (1.5 times more) within the BMP-2 conjugated scaffolds compared with the untreated PCL scaffolds at 4 weeks in vitro [Fig. 2(B)]. Cells proliferated equally well on both scaffold groups as there was no significant difference in DNA content after 4 weeks in vitro [Fig. 2(A)]. This implies that BMP-2 conjugation does not affect the cell proliferation yet it promotes cartilage matrix formation and retention within the scaffold pore space in vitro. The mRNA expression [Fig. 2(C)] measured at 4 weeks in vitro showed that BMP-2 conjugated PCL scaffolds promoted significantly less de-differentiation indicated by lower type 1 collagen expression and also a higher collagen type 2 to collagen type 1 gene expression ratio (col2/col1). The col2/col1 ratio, known as chondrogenic "differentiation index, ${ }^{19}$ " is indicative of more chondrogenic differentiation for cells seeded on the BMP-2 conjugated PCL scaffolds.

However, there was significantly lower aggrecan expression and relatively higher matrix metalloproteinase 13 (MMP-13) expressions for the BMP-2 conjugated group. This may indicate a trend toward endochondral ossification, as MMP-13 is produced by chondrocytes that are possibly 
A
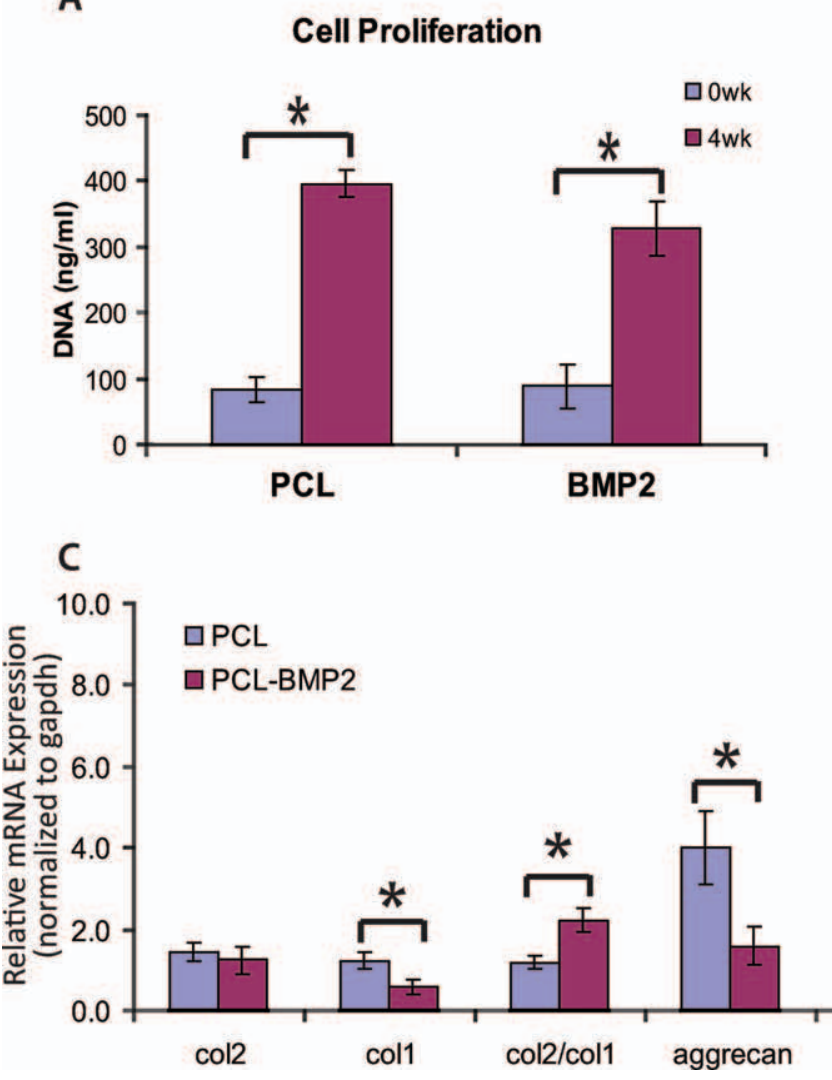

B

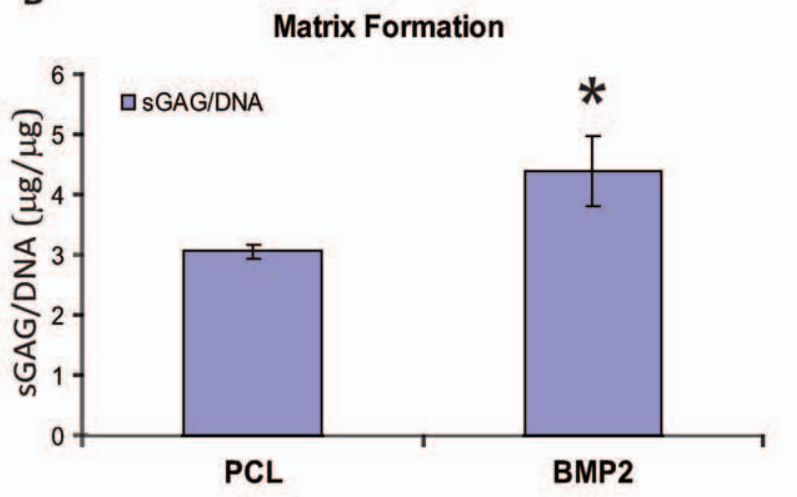

FIGURE 2. (A) the amount of DNA was quantified to observe the cell proliferation from 0 week ( 1 day) to 4 weeks in vitro. (B) The amount of sGAG per DNA was quantified to measure the matrix formation at 4 weeks in vitro. (C) The mRNA expression for cartilage matrix synthesis(col2, col1, col2/col1, and aggrecan), cartilage matrix degradation- (MMP13 and MMP3), hypertrophy- (col10), and bone relevant genes (osteopontin and osteocalcin) was quantified. [Color figure can be viewed in the online issue, which is available at wileyonlinelibrary.com.]

degrading collagen and aggrecan in the cartilage matrix. In spite of the differences in monolayer versus 3D culture conditions and cell sources from different species, Krawczak et al. ${ }^{20}$ also reported similar results as our study that the expression of MMP-13 was upregulated in 5- and 8-week in vitro monolayer cultures of rabbit chondrocytes with direct BMP-2 treatments and suggested that the elevated MMP-13 levels are due to the modulation of chondrocytes differentiation and loss of chondrocytes stability. ${ }^{21-23}$ Furthermore, the significantly higher type 10 collagen expression along with relatively (but not significantly) higher osteopontin expression for the BMP-2 group may also suggest that BMP-2 was accelerating chondrocytes maturation leading to endochondral ossification. Histological examination of both groups [Fig. 3(A)] corresponded to the quantitative cartilage matrix formation data [Fig. 2(B)] supporting the conjecture that BMP-2 conjugated scaffolds promoted cartilage matrix within pore spaces. The BMP-2 group had intense Safranin-O (Saf-0) staining within the scaffold pores, indicative of cartilage matrix, in addition to the presence of round shape lacunae consistent with chondrocytes. Histomorphometric quantification of Saf-O staining demonstrated that both total staining and straining within the scaffold pores only were higher in the BMP-2 group [Fig. 3(B)].

In vitro results clearly demonstrated that conjugated BMP-2 increased cartilage matrix production by chondro- cytes, but also upregulated genes associated with chondrocyte hypertrophy and endochondral ossification. The question is whether the in vitro gene expression results portend that controlled BMP-2 delivery to chondrocytes will induce hypertrophy and increased calcified matrix formation through endochondral ossification. This question was rigorously investigated by implanting chondrocytes seeded on both BMP-2 conjugated PCL and untreated PCL scaffolds for 5 weeks in a highly vascularized subcutaneous environment after 6 weeks of in vitro culture.

Histological examination after 5 weeks in vivo, after 6 weeks in vitro (11 weeks total) demonstrated significantly enhanced Saf-O staining within the scaffold pores for the BMP-2 conjugation group compared with the untreated PCL group [Figs. 3(A) and 4(A): Saf-O/fast green (FG) longitudinal staining]. Furthermore, the presence of chondrocytic cells with round phenotype was more vivid around the inner pore areas. It was obvious that cartilage matrix was better maintained within the center pore space for BMP-2 group where the less nutrients may have been available [Fig. 4(A): Saf-0/FG white lined box]. Like the in vitro study, histomorphometry confirmed that both total and the inner Saf-0 stained area (pore space only) for the BMP-2 conjugated group were significantly higher than nontreated group [Fig. 4(B)]. 
A

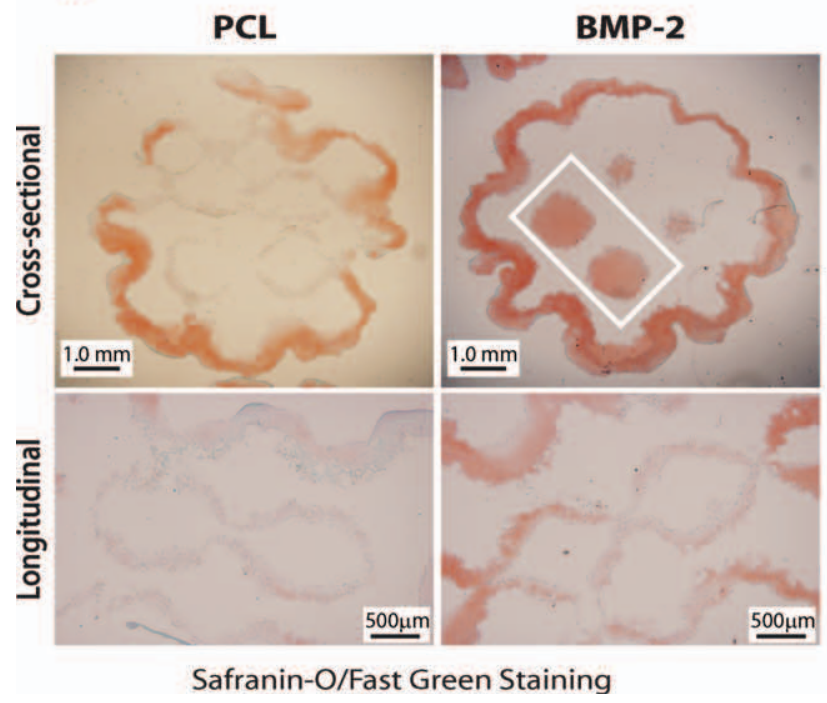

B

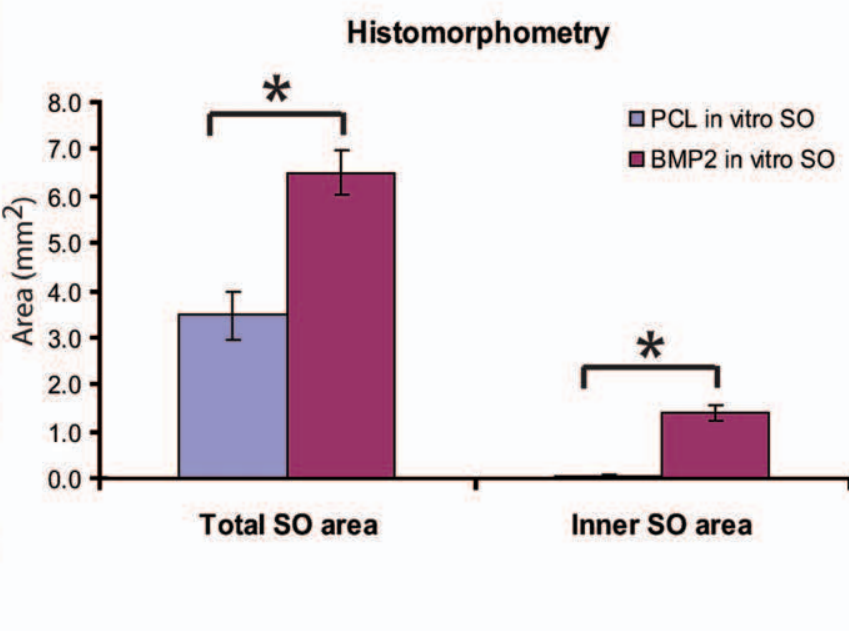

FIGURE 3. In vitro Safranin-O/FG histological examination and semiquantitative histomorphometry demonstrated a comprehensive picture of the protective role of conjugated BMP-2 on chondrogenesis supporting the quantification of matrix formation [Fig. 2(B)]. [Color figure can be viewed in the online issue, which is available at wileyonlinelibrary.com.]

On the outer edged of both scaffold groups' hematoxylin and eosin (H\&E) images [Fig. 4(A)], the BMP-2 treated group presented more and darker stained areas, suggesting areas of both bone and bone marrow [Fig. 4(A): H\&E arrows]. Bone formation was then further characterized via micro-computed tomography (CT). Micro-CT analysis (Fig. 5), however, indicated that there was no significant difference in bone formation in terms of total bone volume, bone mineral content, and tissue mineral content between untreated PCL versus BMP-2 conjugated group. Furthermore, there was no bone ingrowth within the scaffold, demonstrating that bone was formed only around or outside of the scaffold.

\section{DISCUSSION}

In this article, we describe the effect of 3D scaffold conjugated BMP-2 on both in vitro and in vivo cartilage formation by seeded porcine chondrocytes. We also investigated whether BMP-2 conjugation would accelerate chondrocyte hypertrophy and subsequent endochondral ossification when chondrocyte seeded scaffolds were implanted in a vascularized subcutaneous in vivo environment. The contradictory roles of BMP-2, on one hand protecting against cartilage degeneration and on the other hand enhancing chondrocyte hypertrophy were recently reviewed by van der Kraan et al. ${ }^{7}$ Both our in vitro and in vivo results suggest that BMP-2 conjugation facilitates cartilage matrix formation and maintenance particularly within the scaffold spherical pore space. The enhanced cartilage matrix production was perhaps related to BMP-2 inhibiting chondrocyte dedifferentiation as indicated by the lower type I collagen mRNA expression. Unlike the results by Grunder et al., ${ }^{24}$ our results did not indicate any enhancement of the expression of type 2 collagen and aggrecan by BMP- 2 which could be due to the difference in how BMP-2 was exposed to cells (i.e., direct contact vs. through alginate beads or the simple addition vs. conjugation of BMP-2). In a gene therapy study, Kaps et al. ${ }^{5}$ showed similar results to ours in that BMP-2 transfected primary bovine articular chondrocytes revealed no alteration in collagen type II gene expression compared with control chondrocytes.

The histological examination from in vitro to in vivo clearly supported the postulate of BMP-2 promoting cartilage matrix production and efficient retention within the scaffolds (Figs. 3 and 4) compared with untreated PCL. However, there was some evidence from the mRNA expression at 4 weeks in vitro, specifically increased type 10 collagen and osteopontin mRNA expression, that BMP-2 may also accelerate chondrocyte hypertrophy, leading to calcified cartilage being produced at the end stage of endochondral ossification. There was bone formation and bone marrow in vivo at the scaffold periphery in both groups, an area where vascularity would be the highest. However, the amount of bone formed was not significantly different between the BMP-2 conjugated and untreated PCL groups, indicating that the presence of conjugated BMP-2 did not increase bone formation. This result demonstrates that increased gene expression for cartilage hypertrophy and endochondral ossification, namely type 10 collagen and osteopontin, did not translate into increased bone formation in this in vivo model. This may be due to the prolonged localization and slow release of our method for conjugating BMP-2 to scaffolds, which prevents BMP-2 protein from diffusing into surrounding cell niches in an unbound state to initiate ossification. ${ }^{17}$ During the several stages of ectopic bone formation possibly derived by BMP, Tsuyama et al..$^{25}$ reported that the induced bone marrow cells were not from undifferentiated mesenchymal cells in situ, but from hematopoietic stem cells circulating in the peripheral blood. Tsuyama's hypothesis is consistent with our results, especially that the highly 
A

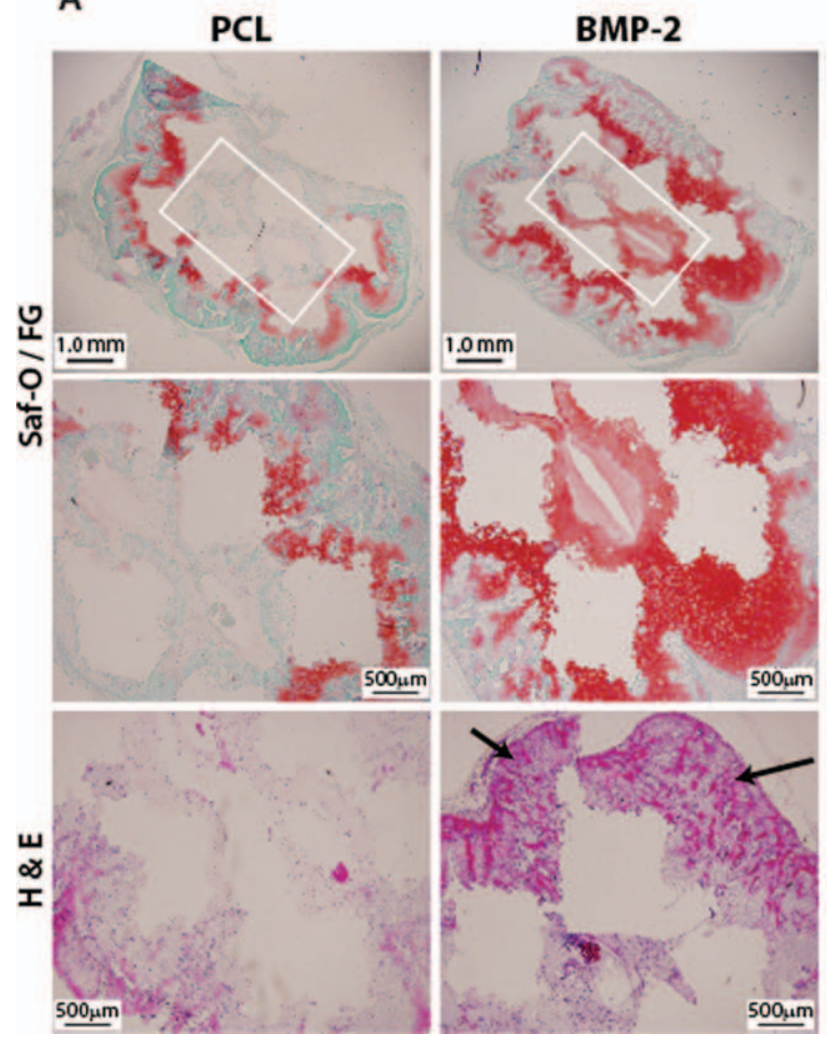

B

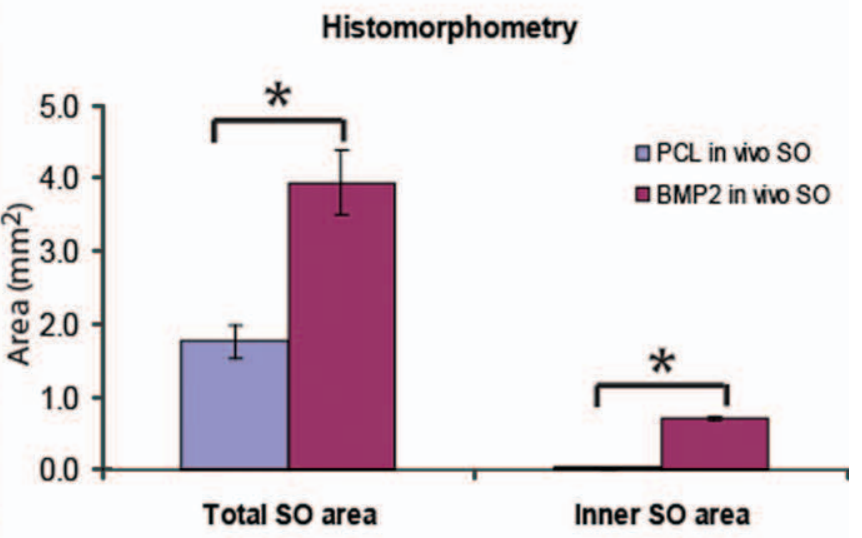

FIGURE 4. In vivo histological examination and histomorphometry ascertained that the protective and promoting role of BMP-2 for chondrogenesis continued from in vitro (Saf-O/FG), but also the future snapshot of hypertrophy predicted by mRNA expression [Fig. 2(C)] was also confirmed by the presence of mature bone marrow at the peripheral scaffold region in H\&E (arrows) staining for BMP-2 group. [Color figure can be viewed in the online issue, which is available at wileyonlinelibrary.com.]

vascular environment on the outside of the scaffold may influence bone regeneration much more than the conjugated BMP2.

Overall, although our in vitro gene expression results suggested the dual protective and degenerative roles of BMP-2 on cartilage in vitro, ${ }^{7}$ our matrix production results in vitro and in vivo suggest that conjugated BMP-2 promoted cartilage formation but not endochondral ossification, even in the highly vascular in vivo microenvironment.

This finding is especially relevant considering that the default fate of chondrocytes or chondrogenically pulsed stem cells is to produce bone in the ectopic model. Oliveira et al. $^{26,27}$ noted that chick embryo chondrocytes cultured for 20 days in vitro produced bone when implanted ectopically from 1 to 5 months on a chitosan scaffold. Liu et al. ${ }^{28}$ chondrogenically pulsed human MSC from 4 to 12 weeks in vitro on PLA scaffolds, and then implanted the constructs from 12 to 24 weeks ectopically in immune-compromised mice. They noted that these constructs supported cartilage formation in vitro, but when implanted subcutaneously the matrix converted to bone if it had been cultured less than 12 weeks in vitro. They suggested that the more immature cartilage matrix had produced VEGF in vitro. However, they did not quantitatively characterized the 3D distribution of bone versus soft tissue in the scaffolds using micro-CT, only through representative histology sections. Thus, it was not possible to discern the relative spatial distribution and amount of mineralized versus soft tissue. However, relative to these results it is possible that the BMP2 conjugated scaffolds produce a mature and stable enough cartilage matrix in vitro to maintain cartilage matrix within the scaffold interior ectopically in vivo.

Although BMP-2 alone clearly enhanced chondrogenesis, several groups ${ }^{29-32}$ have proposed that BMP-2 and TGFbeta1 synergistically and interactively work together for chondrogenesis such that BMP-2 promotes chondrocytes differentiation, whereas TGF-beta1 modulates the chondrocytes maturation and terminal differentiation led by BMP-2. Furthermore, Liu et al. ${ }^{33}$ documented that multiple factors currently used for chondroinduction (including TGF- $\beta 1$, IGF1 , and BMP-2) were present in the supernatant of chondrocyte-engineered constructs and all of these factors were required for initiating chondrogenic differentiation. On the basis of these studies, it would be interesting to conjugate both BMP-2 and TGF-beta1 to observe their effects on chondrogenesis in vitro and in vivo. Indeed, the method of presenting BMP-2 and other growth factors (i.e., conjugation/ tightly bound vs. freely diffusible) is likely to have a significant effect on how these factors influence cell behavior.

Strictly based on this study, our results suggest that chemically conjugated BMP-2 enhanced cartilage matrix synthesis in vitro as seen by the increased sGAG formation, 
A

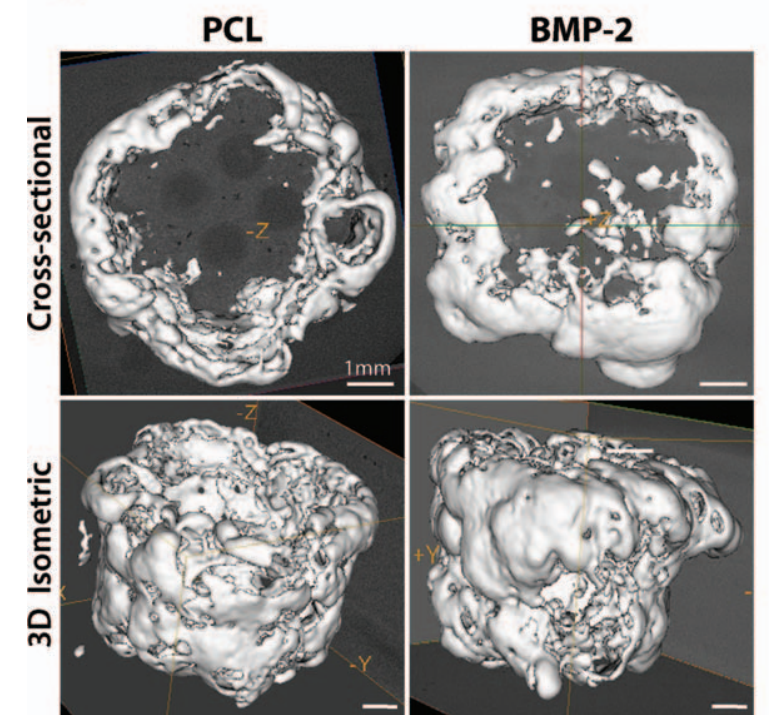

B

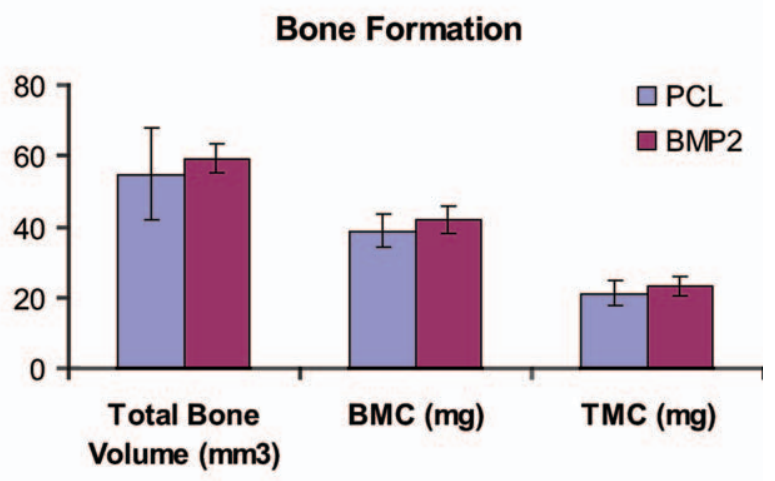

FIGURE 5. In vivo micro-CT images of bone formation for both groups (A) and the corresponding quantitative data (B) clearly presented that the bone formation was not necessarily affected by the presence of BMP-2 directly. There was no significant difference in endochondral ossification for BMP-2 group compared with untreated PCL group. [Color figure can be viewed in the online issue, which is available at wileyonlinelibrary.com.]

more intense sGAG staining, and gene expression and this enhanced chondrogenesis continued in vivo probably due to the prolonged retention of BMP-2. BMP-2 conjugation also upregulated genes associated with cartilage hypertrophy (col 10) and bone turnover formation in vitro (osteopontin), giving some indication of an increased tendency toward endochondral ossification. However, the calcified cartilage resulting from in vivo proteins associated with this gene expression was not found to a significantly higher degree than the control scaffold and it was formed only on the outside of the scaffold. Furthermore, the BMP-2 induced cartilage was still maintained in the interior pores of the scaffold in vivo. This would suggest that BMP-2 does not promote cartilage hypertropy/endochondral ossification to the same extent it promotes cartilage formation. In this case, the bone formation on the outside of the scaffolds in both groups was likely due to the highly vascularized environment on the outside of the scaffold, not particularly due to the presence of conjugated BMP-2. Even though there are some studies suggesting that BMP-2 causes more ossification, these conclusions were mainly based on gene expression, ${ }^{20,34}$ not on the quantification of actual bone matrix formation in vivo. Our study is the first to quantitatively measure both bone and cartilage formation in vitro and in vivo and compare it to previous gene expression. Our study also suggests that there is a desired dose of conjugated BMP-2 that would benefit cartilage regeneration without undesired cartilage calcification and ossification as long as an appropriate and chondrogenically favorable microenvironment is provided (i.e., actual joint defect site instead of highly vascular ectopic site).

Still, one could postulate that our results do not rule out the ability of conjugated BMP-2 to promote both cartilage formation followed by hypertrophy/ossification, if the pro- tein is continuously around for uptake by the chondrocytes. However, there is only a limited amount of tightly bound BMP-2 in the conjugation system. This limited amount of bound BMP-2 may have been taken up by the chondrocytes to a large degree after 6 weeks in vitro. Thus, there may not have been a significant amount of BMP-2 left to affect the seeded or host cells more than the control group in vivo implantation. This postulate could be tested in experiments where a BMP-2 conjugated group with chondrocytes and a control group with chondrocytes are implanted subcutaneously without any in vitro culture to see if the BMP-2 conjugated group makes more bone and has a direct impact on endochondral ossification in vivo.

In conclusion, the tightly bound form of conjugated BMP-2 definitely promoted cartilage matrix production with porcine chondrocytes in an in vitro environment. Furthermore, this enhanced cartilage matrix was protected and maintained in the scaffold interior in a highly vascular in vivo environment. Although there was evidence through gene expression that BMP2 pushed chondrocytes toward hypertrophy and endochondral ossification in vitro, the resultant in vivo bone matrix formation only occurred on the scaffold periphery and was not higher than the control group. Therefore, it may be that BMP-2 must be continuously available to the chondrocytes (even in a highly vascularized environment) to drive more enhanced endochondral ossification after initial enhancement of cartilage matrix production.

\section{MATERIALS AND METHODS}

\section{Scaffold preparation}

3D cylindrical PCL scaffolds $(6.35 \mathrm{~mm}$ diameter, $4 \mathrm{~mm}$ height, $1.62 \mathrm{~mm}$ spherical pores with $550 \mu \mathrm{m}$ internecking pores, $44 \%$ porosity, $100 \%$ interconnectivity) were designed using custom Interactive Data Language ${ }^{\mathrm{TM}}$ programs (IDL; 
Research Systems, Inc.) and the designed scaffolds were manufactured by a laser sintering machine from an .STL file (Fig. 1). The chemically conjugated BMP-2-PCL scaffolds were prepared using the same aminolysis and crosslinking method described previously. ${ }^{17}$ All scaffolds in the BMP-2 group were conjugated with $20 \mu \mathrm{g}$ BMP-2 protein. All the scaffolds were sterilized in sterile $70 \%$ ethanol for $45 \mathrm{~min}$ then briefly rinsed with PBS three times followed by immersion in serum free media before cell seeding.

\section{Cell isolation}

For cell isolation, primary porcine chondrocytes were isolated from the full depth of domestic pig joints as previously described. ${ }^{35}$ Cartilage slices were extracted aseptically within $3 \mathrm{~h}$ of slaughter. Chondrocytes were isolated using a solution of $1 \mathrm{mg} / \mathrm{mL}$ collagenase II (Sigma, St. Louis, MO) in Dulbecco's modified Eagle medium (DMEM) containing $500 \mathrm{U} / \mathrm{mL}$ penicillin and $500 \mathrm{mg} / \mathrm{mL}$ streptomycin $(\mathrm{P} / \mathrm{S})$, $100 \mathrm{U} / \mathrm{mL}$ kanamycin, and 1:500 amphotericin B (Fungizone, Gibco, Carlsbad, CA). The tissue was digested for $5 \mathrm{~h}$ at $37^{\circ} \mathrm{C}$ with gentle agitation. The cell suspension was filtered through a nylon sieve $(70-\mathrm{mm}$ pore size) and the centrifuged cell pellets were resuspended in basal culture medium [DMEM, 10\% fetal bovine serum (FBS), 1\% P/S, Gibco] supplemented with $50 \mathrm{mg} / \mathrm{mL} 2$-phospho-L-ascorbic acid (Sigma) and plated onto tissue culture flasks. Cells were allowed to adhere for $\sim 24 \mathrm{~h}$ then trypsinized and used for in vitro culture.

\section{In vitro cell culture and seeding}

The isolated primary porcine chondrocytes were seeded onto scaffolds and cultured in vitro as previously described with some modification. ${ }^{36-38}$ Cell suspension was added dropwise on top of the scaffold at a density of $1 \times 10^{6}$ cells/scaffold in $60 \mu \mathrm{L}$ culture medium to allow for initial cell attachment in a 24 ultra-low attachment well plate. After $30 \mathrm{~min}, 1.5 \mathrm{~mL}$ of chondrogenic media [basal medium (DMEM, 10\% FBS, 1\% P/S, Gibco) supplemented with $50 \mathrm{mg} / \mathrm{mL}$ 2-phospho-L-ascorbic acid (Sigma), $0.4 \mathrm{mM}$ proline (Sigma), $5 \mathrm{mg} / \mathrm{mL}$ insulin (Gibco), and $0.1 \mathrm{mM}$ nonessential amino acids (Gibco)] was added to each well and further cultured for 4 weeks in vitro or precultured 6 weeks in vitro followed by 5 weeks in vivo in a mouse subcutaneous implantation model. Cell culture was maintained in a water-jacket incubator equilibrated with $5 \% \mathrm{CO}_{2}$ at $37^{\circ} \mathrm{C}$. An orbital shaker was used for in vitro culture and both in vitro and in vivo studies followed the previously established protocols. ${ }^{37,38}$ Four to five scaffolds per group were implanted in vivo subcutaneously.

\section{In vivo implantation}

Samples were cultured in chondrogenic medium for 6 weeks in vitro and cartilage tissue grown scaffolds subsequently implanted into immunodeficient mice for 5 weeks (6- to 8-week-old; N:NIH-bg-nu-xid, Charles River, Wilmington, MA) following previously protocols. ${ }^{35,38}$ Four dorsal subcutaneous pockets were created by blunt dissection and scaffolds from each group were placed in each pouch and in a different pouch location in each mouse. The animals were housed in groups with free access to food and water and scarified after 5 weeks in vivo (total of 11 week culture and implantation) for evaluation. Before implantation, absence of mineralized tissue was confirmed via X-ray. After 5 weeks, implants were removed and processed histologically.

SGAG and DNA quantification. The amount of SGAG and DNA per scaffold $[N=4-5 /$ group at 0 ( 1 day) and 4 weeks in vitro] were determined by using a dimethylmethylene blue (DMMB) assay, Hoechst 33258 dye and a fluorometer, respectively, as previously described..$^{36-38}$ At 0 (1 day) and 4 weeks in vitro, scaffolds at each time point were removed from culture, finely chopped, and placed immediately into $1 \mathrm{~mL}$ of preprepared papain solution [papain (10 units/mg: Sigma Aldrich \#P4762), $1 \times$ PBS, $5 \mathrm{mM}$ cysteine HCL, $5 \mathrm{mM}$ EDTA, $\mathrm{pH}=6.0$; mixed for $2 \mathrm{~h}$ at $37^{\circ} \mathrm{C}$ then filtered] digested for less than $24 \mathrm{~h}$ at $60^{\circ} \mathrm{C} .10 \mu \mathrm{L}$ of the digested tissue-scaffold solution was mixed with $200 \mu \mathrm{L}$ of DMMB reagent and absorbance was read on a plate reader (MultiSkan Spectrum, Thermo, Waltham, MA) at $525 \mathrm{~nm}$. A standard curve was established from chondroitin 6-sulfate from shark (Sigma, C4384) to compare absorbance for samples. ${ }^{39,40}$ The total sGAG were normalized by DNA content which was measured using Hoechst dye 33258 methods (Sigma, \#DNAQF). Again, $10 \mu \mathrm{L}$ digested sample was added to $200 \mu \mathrm{L}$ preprepared Hoechst solution and read with excitation at $355 \mathrm{~nm}$ and emission at $460 \mathrm{~nm}$ (Fluoroskan Ascent FL, Thermo, Waltham, MA) in a 96 well plate. Readings were compared with standard curves made from calf thymus DNA (Sigma, \#DNA-QF). ${ }^{41}$

Quantitative polymerase chain reaction (qtPCR). Chondrocytic differentiation marker genes (type 2 collagen, type $2 / 1$ collagen ratio and aggrecan), de-differentiation marker gene (type 1 collagen), terminal differentiation gene (type 10 collagen), matrix degradation indicator genes (matrix MMP-3, MMP-13), bone formation and bone turnover indicator genes (osteopontin and osteocalcin, respectively) and glyceraldehyde-3-phosphate dehydrogenase (GAPDH) gene expression were determined by qtPCR using a Gene Amp 7700 sequence detection system (Applied Biosystems, Foster City, CA) for scaffolds $(N=3-4 /$ group) cultured 4 weeks in vitro using previously established protocols. ${ }^{37,42}$ The quantity of gene expression was calculated with standard samples and normalized with GAPDH and the already readymade porcine primers were obtained from Applied Biosystems.

Histological examination and histomorphometry. Constructs ( $N=4-5 /$ group) at each time point in vitro and in vivo were fixed in $10 \%$ buffered formalin overnight, dehydrated with a series of graded ethanol, and embedded in paraffin. Tissue sections were stained with $\mathrm{H} \& \mathrm{E}$ or Saf-O/FG counterstaining for scaffolds to assess cell and tissue distribution, cell morphology and sGAG production at the Histology Core of the University of Michigan Dental School. The cross-sectional and longitudinal views of eight to 10 slides 
(two sections/slide) were obtained from the center of each scaffold. ${ }^{38,42}$ Histomorphometry and image analysis was performed on Saf-O stained slides $(N=8-10 /$ group; total and inner Saf-O stained area only) using ImageJ software.

\section{Micro-CT analysis}

The amount of bone for in vivo samples ( $N=4-5 /$ group) was quantified by a MS-130 high resolution Micro-CT Scanner (GE Medical Systems, Toronto, CAN) with $75 \mathrm{kV}, 75 \mathrm{~mA}$, and an aluminum filter. The scanned images were reconstructed and analyzed using Microview software (GE Healthcare) with regions of interest chosen to contain the entire scaffold. ${ }^{43}$

\section{Statistical analysis}

Data are expressed as mean \pm standard deviation. The statistical significance among different groups or time points was calculated using $t$-test using SPSS software. Data were taken to be significant when a $p$-value of 0.05 or less was obtained.

\section{ACKNOWLEDGMENTS}

The authors thank Colleen Flanagan for the scaffold fabrication, Steve Goldstein's ORL lab at University of Michigan for sharing the micro-CT equipment, and Chris Strayhorn for histology.

\section{REFERENCES}

1. Emans PJ, van Rhijn LW, Welting TJ, Cremers A, Wijnands $N$, Spaapen F, Voncken JW, Shastri VP. Autologous engineering of cartilage. Proc Natl Acad Sci U S A 2010;107:3418-3423.

2. Oshin AO, Stewart MC. The role of bone morphogenetic proteins in articular cartilage development, homeostasis and repair. Vet Comp Orthop Traumatol 2007;20:151-158.

3. Yoon BS, Lyons KM. Multiple functions of BMPs in chondrogenesis. J Cell Biochem 2004;93:93-103.

4. Issack PS, DiCesare PE. Recent advances toward the clinical application of bone morphogenetic proteins in bone and cartilage repair. Am J Orthop (Belle Mead NJ) 2003;32:429-436.

5. Kaps C, Bramlage C, Smolian H, Haisch A, Ungethum U, Burmester GR, Sittinger M, Gross G, Haupl T. Bone morphogenetic proteins promote cartilage differentiation and protect engineered artificial cartilage from fibroblast invasion and destruction. Arthritis Rheum 2002;46:149-162.

6. Nawata M, Wakitani S, Nakaya H, Tanigami A, Seki T, Nakamura Y, Saito N, Sano K, Hidaka E, Takaoka K. Use of bone morphogenetic protein 2 and diffusion chambers to engineer cartilage tissue for the repair of defects in articular cartilage. Arthritis Rheum 2005;52:155-163.

7. van der Kraan PM, Davidson EN, van den Berg WB. Bone morphogenetic proteins and articular cartilage: To serve and protect or a wolf in sheep clothing's? Osteoarthritis Cartilage 2010;18:735-741.

8. Claus S, Aubert-Foucher E, Demoor M, Camuzeaux B, Paumier A, Piperno M, Damour O, Duterque-Coquillaud M, Galera P, MalleinGerin F. Chronic exposure of bone morphogenetic protein-2 favors chondrogenic expression in human articular chondrocytes amplified in monolayer cultures. J Cell Biochem 2010;111:1642-1651.

9. Brown KV, Li B, Guda T, Perrien DS, Guelcher S, Wenke JC. Improving bone formation in a rat femur segmental defect by controlling BMP-2 release. Tissue Eng Part A 2011;17:1735-1746.

10. Geiger $M, L i R H$, Friess W. Collagen sponges for bone regeneration with rhBMP-2. Adv Drug Deliv Rev 2003;55:1613-1629.

11. Lee J, Choi WI, Tae G, Kim YH, Kang SS, Kim SE, Kim SH, Jung $Y, \mathrm{Kim} \mathrm{SH}$. Enhanced regeneration of the ligament-bone interface using a poly(L-lactide-co-epsilon-caprolactone) scaffold with local delivery of cells/BMP-2 using a heparin-based hydrogel. Acta Biomater 2011:7:244-257.
12. Patterson J, Siew R, Herring SW, Lin AS, Guldberg R, Stayton PS, Hyaluronic acid hydrogels with controlled degradation properties for oriented bone regeneration. Biomaterials 2010;31:6772-6781.

13. Lopiz-Morales Y, Abarrategi A, Ramos V, Moreno-Vicente C, Lopez-Duran L, Lopez-Lacomba JL, Marco F. In vivo comparison of the effects of RHBMP-2 and RHBMP-4 in osteochondral tissue regeneration. Eur Cell Mater 2010;20:367-378.

14. Morisue $H$, Matsumoto $M$, Chiba $K$, Matsumoto $H$, Toyama $Y$, Aizawa M, Kanzawa N, Fujimi TJ, Uchida H, Okada I. A novel hydroxyapatite fiber mesh as a carrier for recombinant human bone morphogenetic protein-2 enhances bone union in rat posterolateral fusion model. Spine (Phila Pa 1976) 2006;31:1194-1200.

15. Gavenis K, Schneider U, Groll J, Schmidt-Rohlfing B. BMP-7loaded PGLA microspheres as a new delivery system for the cultivation of human chondrocytes in a collagen type I gel: The common nude mouse model. Int J Artif Organs 2010;33:45-53.

16. Patel ZS, Yamamoto M, Ueda H, Tabata Y, Mikos AG. Biodegradable gelatin microparticles as delivery systems for the controlled release of bone morphogenetic protein-2. Acta Biomater 2008;4:1126-1138.

17. Zhang $\mathrm{H}$, Migneco $\mathrm{F}$, Lin $\mathrm{CY}$, Hollister SJ. Chemically-conjugated bone morphogenetic protein-2 on three-dimensional polycaprolactone scaffolds stimulates osteogenic activity in bone marrow stromal cells. Tissue Eng Part A 2010;16:3441-3448.

18. Reddi AH. Morphogenesis and tissue engineering of bone and cartilage: Inductive signals, stem cells, and biomimetic biomaterials. Tissue Eng 2000;6:351-359.

19. Martin I, Jakob M, Schafer D, Dick W, Spagnoli G, Heberer M Quantitative analysis of gene expression in human articular cartilage from normal and osteoarthritic joints. Osteoarthritis Cartilage 2001;9:112-118.

20. Krawczak DA, Westendorf JJ, Carlson CS, Lewis JL. Influence of bone morphogenetic protein-2 on the extracellular matrix, material properties, and gene expression of long-term articular chondrocyte cultures: Loss of chondrocyte stability. Tissue Eng Part A 2009;15:1247-1255.

21. Burrage PS, Mix KS, Brinckerhoff CE. Matrix metalloproteinases: Role in arthritis. Front Biosci 2006;11:529-543.

22. Malemud CJ. Matrix metalloproteinases: Role in skeletal development and growth plate disorders. Front Biosci 2006;11:1702-1715.

23. Cawston TE, Wilson AJ. Understanding the role of tissue degrading enzymes and their inhibitors in development and disease. Best Pract Res Clin Rheumatol 2006;20:983-1002.

24. Grunder T, Gaissmaier C, Fritz J, Stoop R, Hortschansky P, Mollenhauer J, Aicher WK. Bone morphogenetic protein (BMP)-2 enhances the expression of type II collagen and aggrecan in chondrocytes embedded in alginate beads. Osteoarthritis Cartilage 2004;12:559-567.

25. Tsuyama K, Takaoka K, Kitamura Y, Ono K. Origin of the marrow cells in bones induced by implantation of osteosarcoma-derived bone-inducing factor in mice. Clin Orthop Relat Res 1983:251-256.

26. Oliveira SM, Amaral IF, Barbosa MA, Teixeira CC. Engineering endochondral bone: In vitro studies. Tissue Eng Part A 2009;15:625-634.

27. Oliveira SM, Mijares DQ, Turner G, Amaral IF, Barbosa MA, Teixeira CC. Engineering endochondral bone: In vivo studies. Tissue Eng Part A 2009;15:635-643.

28. Liu K, Zhou GD, Liu W, Zhang WJ, Cui L, Liu X, Liu TY, Cao Y. The dependence of in vivo stable ectopic chondrogenesis by human mesenchymal stem cells on chondrogenic differentiation in vitro. Biomaterials 2008;29:2183-2192.

29. Toh WS, Liu H, Heng BC, Rufaihah AJ, Ye CP, Cao T. Combined effects of TGFbeta1 and BMP2 in serum-free chondrogenic differentiation of mesenchymal stem cells induced hyaline-like cartilage formation. Growth Factors 2005;23:313-321.

30. Hanada K, Solchaga LA, Caplan Al, Hering TM, Goldberg VM, Yoo JU, Johnstone B. BMP-2 induction and TGF-beta 1 modulation of rat periosteal cell chondrogenesis. J Cell Biochem 2001;81:284-294.

31. Kim MK, Niyibizi C. Interaction of TGF-beta1 and rhBMP-2 on human bone marrow stromal cells cultured in collagen gel matrix. Yonsei Med J 2001;42:338-344.

32. van Beuningen $\mathrm{HM}$, Glansbeek $\mathrm{HL}$, van der Kraan PM, van den Berg WB. Differential effects of local application of BMP-2 or TGF- 
beta 1 on both articular cartilage composition and osteophyte formation. Osteoarthritis Cartilage 1998;6:306-317.

33. Liu X, Sun H, Yan D, Zhang L, Lv X, Liu T, Zhang W, Liu W, Cao $Y$, Zhou G. In vivo ectopic chondrogenesis of BMSCs directed by mature chondrocytes. Biomaterials 2010;31:9406-9414.

34. Majumdar MK, Chockalingam PS, Bhat RA, Sheldon R, Keohan C, Blanchet T, Glasson S, Morris EA. Immortalized mouse articular cartilage cell lines retain chondrocyte phenotype and respond to both anabolic factor BMP-2 and pro-inflammatory factor IL-1. J Cell Physiol 2008;215:68-76.

35. Liao E, Yaszemski M, Krebsbach P, Hollister S. Tissue-engineered cartilage constructs using composite hyaluronic acid/collagen hydrogels and designed poly(propylene fumarate) scaffolds. Tissue Eng 2007;13:537-550.

36. Jeong CG, Hollister SJ. Mechanical and biochemical assessments of three-dimensional poly(1,8-octanediol-co-citrate) scaffold pore shape and permeability effects on in vitro chondrogenesis using primary chondrocytes. Tissue Eng Part A 2010;16:3759-3768.

37. Jeong CG, Hollister SJ. A comparison of the influence of material on in vitro cartilage tissue engineering with $\mathrm{PCL}, \mathrm{PGS}$, and $\mathrm{POC}$ 3D scaffold architecture seeded with chondrocytes. Biomaterials 2010;31:4304-4312.
38. Jeong CG, Zhang $\mathrm{H}$, Hollister SJ. Three-dimensional poly(1,8octanediol-co-citrate) scaffold pore shape and permeability effects on sub-cutaneous in vivo chondrogenesis using primary chondrocytes. Acta Biomater 2011;7:505-514.

39. Farndale RW, Buttle DJ, Barrett AJ. Improved quantitation and discrimination of sulphated glycosaminoglycans by use of dimethylmethylene blue. Biochem Biophys Acta 1986;883:173-177.

40. Chandrasekhar S, Esterman MA, Hoffman HA. Microdetermination of proteoglycans and glycosaminoglycans in the presence of guanidine hydrochloride. Anal Biochem 1987;161:103-108.

41. Kim YJ, Sah RL, Doong JY, Grodzinsky AJ. Fluorometric assay of DNA in cartilage explants using Hoechst 33258. Anal Biochem 1988;174:168-176.

42. Jeong CG, Hollister SJ. Mechanical, permeability, and degradation properties of 3D designed poly(1,8 octanediol-co-citrate)($\mathrm{POC})$ scaffolds for soft tissue engineering. J Biomed Mater Res B Appl Biomater 2010;93:141-149.

43. Saito E, Kang H, Taboas JM, Diggs A, Flanagan CL, Hollister SJ. Experimental and computational characterization of designed and fabricated 50:50 PLGA porous scaffolds for human trabecular bone applications. J Mater Sci Mater Med 2010;21:2371-2383. 